\title{
Cardiopulmonary Bypass in Ewe's Fetus: Advances and Setbacks in Our Learning Curve
}

\author{
A. Crucean, *† B. Murzı,* A. Giorgi,* S. Burchiellı, $¥$ M. Trivella, $\neq$ and F. Coceanił
}

Fetal cardiac surgery represents a surgical challenge and several centers are attempting to establish a suitable methodology in animals. We present our experience with extracorporeal bypass procedures in preterm and term sheep fetuses. Twenty-two fetuses (103-139 days gestation, mean 115 days gestation) underwent a 1-hour period of right heart-topulmonary artery extracorporeal circulation followed by 1 hour of observation. Animals were divided into group 1 and group 2, according to gestational age (above and below 0.85 ). Three pumps were used: centrifugal without (group 1) reservoir, centrifugal with (group 2) reservoir, and roller with reservoir (group 2). Experiments were completed in $75 \%$ of fetuses in group 1 and in $37 \%$ of fetuses in group 2. Bleeding was the main cause of failure, especially for group 2. A slow deterioration of blood gas status was noted in group 1, while this trend could be partially reversed in group 2 with corrective measures. Complete heart bypass could not be achieved in either group, and residual fluctuations in arterial pressure were observed. During bypass, body temperature decreased more in group 2 than in group 1 . We conclude that cardiac bypass is feasible over a short period in near-term fetuses. A successful outcome may also be obtained in younger fetuses, but better measures need to be implemented for the prevention of surgical bleeding. ASAIO Journal 2005; 51:649-653.

uring the last decade, we have witnessed a marked improvement in prenatal diagnosis of cardiac malformations, especially with high-risk pregnancies where echocardiography may detect complex malformations as early as 12-16 weeks of gestation. ${ }^{1,2}$ However, prenatal diagnosis has not translated into any substantive improvement in postnatal survival ${ }^{2-4}$ and has simply ensured better surveillance of pregnancy in specialized centers. Ultimately, at least a third of these families opt to terminate a pregnancy, despite the counseling offered by multispecialized teams. ${ }^{5}$ In reaching this decision, parents are guided not only by ethical issues, but also by the lack of a valid alternative based on long-term follow-up and the quality of life for children undergoing complex cardiac neonatal repair.

From *`G Pasquinucci' Hospital for Cardiac Surgery, Massa, Italy; \#Institute of Clinical Physiology, National Research Council, Pisa, Italy; and +Scuola Superiore 'S. Anna,' Pisa, Italy.

Submitted for consideration and accepted in revised form June 2005.

Presented in part at the First International Conference on Pediatric Mechanical Circulatory Support Systems and Pediatric Cardiopulmonary Perfusion, May 19-22, Hershey, PA, USA.

Reprint Requests: Dr. Adrian Crucean, Pediatric Cardiac Surgery, Pediatric Cardiac Hospital, Massa, Via Aurelia Sud, 54100, MS, Italy; e-mail: disor99@yahoo.com

DOI: 10.1097/01.mat.0000178048.90243.e1
Complex, severe pathologies would benefit from prenatal diagnosis and intervention considering that the "form-followsflow" concept predicts cardiac morphogenesis to be conditioned by mechanical solicitations.5,6 Indeed, some cardiac malformations evolve through fetal life, progressing as a consequence of the abnormal transvalvular pattern flow and modified intracardiac pressures. Accordingly, antenatal detection of aortic/pulmonary stenosis would warrant percutaneous or open-chest fetal interventions to prevent an evolution towards hypoplasia of either cardiac cavities or great arteries.

Worldwide experience in fetal percutaneous valvuloplasty is limited and largely disappointing, ${ }^{7-10}$ and the surgical approach is still at an experimental stage in animals. ${ }^{11,12}$ Troublesome issues include intervention at an advanced gestational age when benefit is not optimal, difficult access to the cardiac outflow sites, persistent cardiac hypoplasia despite some favorable short-term results, intrauterine growth retardation, premature delivery, fetal death, and placental hypoperfusion. From a technical standpoint, the major challenges to cardiac surgery, specifically in younger fetuses, relate to the necessity of designing a special bypass setup and the danger of fetoplacental impairment. ${ }^{12}$

The main objective of this study was to examine the feasibility of surgery with fetal bypass, specifically at an early stage of gestation, using different hemodynamic pumps. Our approach paralleled that being used in the neonatal cardiac surgery unit.

\section{Materials and Methods}

Twenty-two time-dated pregnant ewes (days gestation: 103139, median 115, mean 117; term 145 days) were anesthetized and monitored for arterial and central venous pressures, electrocardiographic features, and arterial blood gas status. Propofol (Diprivan) and nitrous oxide were used for induction/ maintenance of anesthesia of the mother. Tiletamin and zolazepam (Zoletil) and succinylcholine chloride (Midarine) were used for the fetus.

The uterus was exposed and the neck blood vessels of the fetus were exposed. Fetal monitoring included arterial and central venous pressures and nasopharyngeal temperature. Continuous recording was made using a Gould - TA 5000 polygraph. Umbilical blood flow was measured in certain experiments with a 4-mm probe coupled to a Triton Dual Flowmeter. Heparin sodium was administered to all ewes (3 $\mathrm{mg} / \mathrm{kg}$ ), but only to certain fetuses $(1 \mathrm{mg} / \mathrm{kg}$ ) depending on the anticoagulation status from anticoagulation time values.

The fetus was prepared for extracorporeal circulation through a median sternotomy and cannulation of the right atrium and main pulmonary trunk (Stockert curved-tip arterial 
and Dideco straight and/or angled-tip venous cannulae). The protocol envisaged a bypass of 1 hour followed by 1 hour of observation. Three different pump devices were used: simple centrifugal (Medtronic), centrifugal + Polystan Safe Micro reservoir, and roller pump (Stockert) + reservoir. In all cases, the circuit was 3/16 inch on the arterial limb and 1/4 inch on the venous limb. Blood gas analysis was performed every 20 minutes in both fetus and sheep. In the event of hypovolemia (mainly due to fetal bleeding) or acid/base imbalance, fluids (Ringer solution) and sodium bicarbonate ( $8.4 \%$ solution) were administered, while the fetus was transfused with maternal blood. The priming solution for the circuit consisted of Ringer solution $(80 \mathrm{ml})$, sodium bicarbonate $8.4 \%(5 \mathrm{ml})$, heparin sodium $(1 \mathrm{mg} / \mathrm{kg})$, and calcium gluconate $(5 \mathrm{ml})$. This was partially replaced at the beginning of bypass with $50 \mathrm{ml}$ maternal blood. All values are presented as means and standard deviations. Statistical significance of the results was assessed with analyses of variance for repeated measurements.

Surgery and protocols were approved by the Animal Care Committee of the Ministry of Health.

\section{Results}

Experiments were completed in 9 cases $(41 \%)$ with fetuses between 105 and 139 days gestation (mean 120 days, median 118 days). Bypass could not be initiated in 6 more cases (27\%), while in $7(32 \%)$ it lasted less than 1 hour.

Considering a cut-off point of 0.75 gestational age (109 days), successful experiments were performed in 25\% (2 cases) of fetuses below this age and in 58\% (7 cases) above that age. A cut-off point of 0.85 (corresponding to 124 days gestation) would lead instead to $37 \%$ and $75 \%$ completed experiments, respectively, below (group 2; 6 animals) and above (group 1; 3 animals) this limit. For each group, we present the results in relation to the following variables: type of pump used, umbilical and pump flows achieved, presence of arterial pressure oscillations, changes in hematocrit, arterial blood gas status and body temperature, and requirements for transfusion or acid/base balance correction.

\section{Complete Experiments: Group 1}

We used a centrifugal head pump for the bypass (all three cases), atrial cannulae measured 3.5 or $4 \mathrm{~mm}$ and venous cannulae were 16 or $18 \mathrm{Fr}$ depending on the calibre of the great vessel. Pump flow varied from 300 to $950 \mathrm{ml} / \mathrm{min}$, the mean value for a stable pump flow (steady conditions) being $633 \pm 256 \mathrm{ml} / \mathrm{min}$.

Although fetal central venous pressure decreased during bypass to $6 \pm 3 \mathrm{~mm} \mathrm{Hg}$ (from $9 \pm 3 \mathrm{~mm} \mathrm{Hg}$ ), the arterial pressure curve maintained its normal oscillations despite a significant reduction of the differential pressure to 4-12 $\mathrm{mm}$ $\mathrm{Hg}$ (mean pressure 52/46 $\pm 5 \mathrm{~mm} \mathrm{Hg}$ ). Prebypass mean fetal arterial pressure was $62 / 50 \pm 9 \mathrm{~mm} \mathrm{Hg}$, whereas the postbypass value was $65 / 45 \pm 4 \mathrm{~mm} \mathrm{Hg}$. Umbilical flow was measured in all experiments and fluctuated between 100 and 220 $\mathrm{ml} / \mathrm{min}$ with a slight decrease during the bypass period (to a mean of $79 \pm 17 \%$ of prebypass flow). The postbypass umbilical flow continued to decrease in two cases to $47 \% \pm 13 \%$ and partially recovered in one further case to $90 \%$ of the prebypass value. Blood gas analysis for the main variables $(\mathrm{pH}$,
Table 1. Arterial Blood Gas Status for Fetuses above 0.85 Gestational Age (Group 1)

\begin{tabular}{cccc}
\hline Time & $\mathrm{pH}$ & $\mathrm{pO}_{2}(\mathrm{~mm} \mathrm{Hg})$ & $\mathrm{pCO}_{2}(\mathrm{~mm} \mathrm{Hg})$ \\
\hline Before CPB & $7.31 \pm 0.05$ & $22.6 \pm 9.1$ & $50.6 \pm 18.3$ \\
During CPB & & & \\
$20 \mathrm{~min}$ & $7.27 \pm 0.05$ & $22.2 \pm 19.1$ & $47.2 \pm 7.5$ \\
$40 \mathrm{~min}$ & $7.24 \pm 0.04$ & $19.8 \pm 8.9$ & $57.3 \pm 19.6$ \\
60 min & $7.21 \pm 0.06$ & $18.9 \pm 9.2$ & $55.5 \pm 8.2$ \\
After CPB & & & \\
20 min & $7.20 \pm 0.05$ & $14.4 \pm 7.8$ & $62 \pm 19.8$ \\
40 min & $7.21 \pm 0.06$ & $14.8 \pm 8.9$ & $66 \pm 12.1$ \\
60 min & $7.18 \pm 0.06$ & $14.6 \pm 9.1$ & $67.1 \pm 9.1$ \\
\hline
\end{tabular}

Values $(n=3)$ are means $\pm S D$ and apply to measurements before, during, and after cardiopulmonary bypass (CPB). In the course of CPB and afterwards, data were obtained at 20-minute intervals.

$\mathrm{pO}_{2}, \mathrm{pCO}_{2}, \mathrm{O}_{2}$ saturation) is given in Table 1. A decrease in oxygenation $\left(\mathrm{pO}_{2}\right)$ with concomitant $\mathrm{pCO}_{2}$ rise and $\mathrm{pH}$ reduction was noted throughout the experiment, but variables were relatively stable in the postbypass period. In only one case did $\mathrm{pH}$ fall below 7.0.

Large changes were noted in hematocrit values, from $34 \% \pm$ $12 \%$ before to $23 \% \pm 11 \%$ during and after bypass. Maternal blood was transfused to the fetus in case of hypovolemia and the mean amount was $80 \pm 15 \mathrm{ml}$. Likewise, the acid/base imbalance was corrected with sodium bicarbonate solution administered, during and after extracorporeal circulation (mean volumes were $28 \pm 2 \mathrm{ml}$ ). Fetal temperature decreased to a minimum of $34^{\circ} \mathrm{C} \pm 0.8^{\circ} \mathrm{C}$ during the preparation period and to $33.2^{\circ} \mathrm{C} \pm 0.9^{\circ} \mathrm{C}$ during the bypass period.

\section{Complete Experiments: Group 2}

In these animals, we used a centrifugal pump (one case), then a centrifugal pump coupled with a venous reservoir (two cases), and a roller pump and reservoir (three cases). Arterial cannulae measured $3 \mathrm{~mm}$ and venous cannulae were 14 or $16 \mathrm{Fr}$ depending on the calibre of the vessels. Pump flow varied from 120 to $700 \mathrm{ml} / \mathrm{min}$ with a mean value for the maximum stable flow of $416 \pm 193 \mathrm{ml} / \mathrm{min}$. Central venous pressure fell during the bypass period from $12.3 \pm 3 \mathrm{~mm} \mathrm{Hg}$ to $8.6 \pm 2.9 \mathrm{~mm} \mathrm{Hg}$, returning to normal values after bypass. Arterial pressure curve maintained its oscillations whatever the type of pump used and despite a reduction of the differential pressure to $6-12 \mathrm{~mm} \mathrm{Hg}$. (mean pressure $57 / 45 \pm 4 \mathrm{~mm} \mathrm{Hg}$ ). Prebypass mean fetal arterial pressure was $70 / 57 \pm 8 \mathrm{~mm} \mathrm{Hg}$, whereas the postbypass value was $61 / 43 \pm 6$ $\mathrm{mm} \mathrm{Hg}$. Umbilical flow was measured in two cases and varied between 140 and $160 \mathrm{ml} / \mathrm{min}$ with a slight decrease to a mean value of $80 \% \pm 5 \%$ of control (prebypass flow). After bypass, it moved upwards to $93 \% \pm 6 \%$ of control (prebypass period). Blood gas analysis for the main variables $\left(\mathrm{pH}, \mathrm{pO}_{2}, \mathrm{pCO}_{2}, \mathrm{O}_{2}\right.$ saturation) is given in Table 2 . A decrease in $\mathrm{pO}_{2}$, with $\mathrm{pCO}_{2}$ rise and $\mathrm{pH}$ adjustment, was noted towards the end of bypass period and in the initial part of the postbypass period. However, partial restoration of the gas status occurred near the end of the experiment. Most animals with this "recovery pattern" belong to group 2 (four animals), whereas only one is found in group 1.

Mean hematocrit varied from $36.2 \% \pm 8 \%$ (prebypass) to $28.7 \% \pm 7 \%$ and $29 \pm 7.1 \%$, respectively, during and after the bypass period. Requirements of blood transfusion (fetus) and 
Table 2. Arterial Blood Gas Status for Fetuses below 0.85 Gestational Age (Group 2)

\begin{tabular}{cccc}
\hline Time & $\mathrm{pH}$ & $\mathrm{pO}_{2}(\mathrm{~mm} \mathrm{Hg})$ & $\mathrm{pCO}_{2}(\mathrm{mmHg})$ \\
\hline Pre CPB & $7.31 \pm 0.09$ & $26.7 \pm 6.5$ & $49.8 \pm 13.1$ \\
During CPB & & & \\
$20 \mathrm{~min}$ & $7.25 \pm 0.05$ & $23.1 \pm 7.1$ & $55 \pm 6.8$ \\
$40 \mathrm{~min}$ & $7.24 \pm 0.05$ & $20.7 \pm 7.3$ & $58.6 \pm 5.6$ \\
$60 \mathrm{~min}$ & $7.23 \pm 0.06$ & $21.1 \pm 15.9$ & $60.5 \pm 4$ \\
Post CPB & & & \\
$20 \mathrm{~min}$ & $7.20 \pm 0.05$ & $20.9 \pm 6.3$ & $67 \pm 14.2$ \\
$40 \mathrm{~min}$ & $7.13 \pm 0.04$ & $17.2 \pm 5.8$ & $79.5 \pm 6.1$ \\
$60 \mathrm{~min}$ & $7.18 \pm 0.05$ & $23 \pm 6.7$ & $54.6 \pm 12.8$ \\
\hline
\end{tabular}

Values $(n=6)$ are means $\pm S D$ and apply to measurements before, during, and after cardiopulmonary bypass (CPB). In the course of CPB and afterwards, data were obtained at 20-minute intervals.

sodium bicarbonate solution were $96 \pm 14 \mathrm{ml}$ and $36 \pm 5 \mathrm{ml}$, respectively. Fetal temperature decreased to a minimum of $35^{\circ} \mathrm{C} \pm 0.9^{\circ} \mathrm{C}$ during the preparation period and to $31.9^{\circ} \mathrm{C} \pm$ $1.9^{\circ} \mathrm{C}$ during bypass period.

\section{Incomplete Experiments}

There was a single death of a ewe due to uncontrollable abdominal bleeding before the start of extracorporeal circulation. All other pregnant sheep were weaned off the ventilator at the end of the experiment.

Bypass was not initiated in one fetus above 0.85 gestational because of persistent bradycardia. No obvious explanation was found for this condition.

For young fetuses ( $<0.85$ gestational age), the main cause of failure to initiate bypass or for its premature interruption was bleeding occurring either diffusely from all tissues (one case) or from the site of atrial cannulation (nine cases). Other causes were severe fetal bradycardia (one case) or thrombosis in the circuit (one case). In total, bypass was not initiated in five cases. In the unsuccessful experiments, we used for bypass a centrifugal head pump (seven cases) or a centrifugal pump coupled with a reservoir (one case).

\section{Discussion}

The original aim of the study was to perform experiments on fetuses at 0.7 gestational age or less using the same approach of a neonatal cardiac surgery unit. Nevertheless, we decided to use first near-term fetuses to identify the major problems of the procedure. Soon it became clear that there are no major technical difficulties with older fetuses (group 1), and bypass could be completed and studied according to protocol. Nevertheless, the tendency to slow deterioration during the bypass period with relative stability through the postbypass period was evident in all animals in group 1 . This was not the case for younger animals (group 2), where the main problem was bleeding and the attendant inability to start or complete the bypass. Bleeding during purse-string construction/tightening or cannulation was mostly caused by the fragility of the atrial tissue. By moving the atrial cannulation site towards the atriocaval junction and by using a smaller needle $(7 / 0$ or $8 / 0$ Prolene), the incidence of failures decreased. Nevertheless, in the small fetuses, only once could the purse-string/cannulation procedure be performed. Any incident requiring hemostasis at the atrial site resulted in intractable bleeding with tearing of the atrial wall, usually close to the atrioventricular groove. In four of the younger cases (103-107 days gestation), atrial tissue was so fragile that it was torn off by the centrifugal pump vortex. Introduction of the gravitational reservoir for venous drainage in both centrifugal and roller head pumps solved this particular problem. In addition, the latter approach presented the advantage of saving blood, by allowing its recirculation from the surgical field through the roller pump. In the end, six experiments (representing $66 \%$ of all completed experiments) were performed on fetuses at 0.7-0.8 gestation. Bleeding was profuse in one case, but we could not identify its cause in an overdose of heparin. Moreover, in one case, the administration of heparin only to the sheep led to formation of thrombi in the circuit (already primed with heparin). From then on, heparin was administered to both ewe and fetus, using a heparinprimed circuit as well.

We avoided using a specially designed intra-axial head pump, as described by Grigioni et al., ${ }^{11}$ because of its cost, lack of availability, and the fact that it is not being used in neonatal surgery. We wished, in fact, to align our experimental approach with current practice. Priming the circuit was possible in this case by using a clear solution that was subsequently replaced in part $(60 \%)$ by fresh maternal blood just before the start of the bypass. In the end, we had the advantage of using a warm, blood-enriched priming solution.

Complete heart bypass similar to that obtained during neonatal extracorporeal circulation with a single atrial cannula was difficult to achieve. Despite the suitable venous cannulae and verification of atrial emptying during the bypass, arterial fluctuations persisted in both groups, albeit in a reduced measure. This finding raised the question of a certain amount of blood bypassing the heart through a patent foramen ovale, the tricuspid valve, a patent ductus arteriosus, or pulmonary arteries/veins. In fetuses at 0.9 gestation, maximum umbilical flow was $200-220 \mathrm{ml} / \mathrm{min}$ with a pump flow of $650-950 \mathrm{ml} / \mathrm{min}$. Central venous pressure was slightly higher in group 2, but arterial oscillations never disappeared, whatever the type of the head pump being used. Venous cannulae were either straight or angled Polystan, ranging from 14 to $18 \mathrm{Fr}$, which in a small infant can easily drain $270-400 \mathrm{ml} / \mathrm{min}$ with a roller pump or $700-1500 \mathrm{ml} / \mathrm{min}$ with a centrifugal pump. Considering the amount of blood being calculated on the basis of body weight or gestational age/weight curve, ${ }^{13}$ the cannulae should have been large enough to accommodate these volumes of flow. Whereas in group 1 these volumes of blood could be drained through a centrifugal pump, the same could not be achieved in group 2 because this type of pump produced atrial bleeding. Success was obtained with the roller pump coupled to a reservoir, but arterial oscillations persisted. Arterial Stockert cannula was 3 or $4 \mathrm{~mm}$ and presented no problems in conveying this kind of flow. Even a venous drainage with two, rather than one, cannulae did not secure a total bypass. In considering possible explanations for this finding, we noted the patent foramen ovale was unusually positioned distally towards inferior vena cava. This particular location, which has been described in the literature, ${ }^{14}$ can impede optimal drainage. For this reason, we inserted the cannula deeper towards the vein. This approach, together with the use of a venous reservoir with a roller head pump, improved 
drainage and provided stability during bypass, but still results were not optimal. Cannulation of the aorta, clamping of the pulmonary trunk proximally to the cannula, and tying of pulmonary branches reduced further arterial pressure oscillations, but did not eliminate them completely. Nevertheless, in fetuses in group 1, we were able to obtain stable pump flows at 250-300 $\mathrm{ml} \cdot \mathrm{kg}^{-1} \cdot \mathrm{min}^{-1}$, which are similar to those cited in the literature with axial-incorporated rotating pumps. For fetuses in group 2, the main obstacle in achieving a greater flow with the centrifugal pump was the risk of atrial tear despite adequate filling. When a roller pump was used, an almost completely collapsed atrium was clearly noted. Nevertheless, arterial oscillations were recorded, albeit with a reduced amplitude. These oscillations might have been due to the pooling of blood in an extrafetal compartment (placenta) or to lack of equilibrium among different fluid compartments in the fetus along with a rapid intracardiac bypass.

The combination of ketamine with benzodiazepine for fetal anesthesia provided satisfactory hemodynamic stability and muscular relaxation. In the two instances in which bypass was not started due to bradycardia, we could not associate this event with the anesthesia. However, a relatively slow cardiac rhythm was noted when first examining the fetus inside the uterus.

The umbilical artery is hard to isolate within the abdomen in young fetuses. For this reason, we opted to record flow from the vessel in the umbilical cord, taking care with gentle manipulation not to cause constriction. Regrettably, the flowmeter in the present probe configuration $(4 \mathrm{~mm})$ proved not to be reliable with the younger fetuses (group 2).

Body temperature homeostasis was a major concern. Through the entire procedure, fetal body warming was assured with an infrared lamp and gauzes soaked in warm saline. Initially, we collected the amniotic fluid (lost through a large hysterotomy) and reintroduced it (after fetal preparation) in the uterus at the beginning of the bypass. We then opted to suture the edges of the uterine incision on the fetal abdominal wall, thus conserving the amniotic fluid. In the end, we noticed that it was possible to match the uterine incision onto the abdominal fetal wall of the fetus without the need of any suture, especially with small fetuses. Nevertheless, with the introduction of a reservoir in the circuit, it was difficult to maintain the temperature through the bypass. External warming of the reservoir solved this problem, and the maximum gradient between prebypass and bypass temperatures did not exceed $3^{\circ} \mathrm{C}$.

Blood gas values were used as an index of placental function because conditions of the mother remained stable throughout the procedure. Although a trend towards deterioration was noted in group 2 initially or throughout the whole bypass, some comments are warranted. A slight improvement in blood gas conditions and in the umbilical flow was observed towards the end of the observation period, even though complete restoration of function was never achieved. However, there is a bias in this observation because active measures were taken to correct, on one hand, fetal hypovolemia with maternal blood and, on the other, maternal and/or fetal acid/base imbalance with sodium bicarbonate solution. In group 1, there was a slow deterioration in the blood gas status, but eventually a stable condition was obtained in the last part of the observation period. With the data available, it is difficult to decide whether better recovery in the older fetuses (group 1) reflected an adaptive mechanism or a more efficient flow through bypass. The use of centifugal (all cases in group 1) versus roller pump (half the cases in group 2) flow is one of the differences between the two groups, and it may account, at least in part, for this finding. There were two cases in group 2 that presented exceptionally high values of oxygenation during the whole procedure. This influenced in an important way mean $\mathrm{pO}_{2}$ values for all group 2 animals (the mean $\mathrm{pO}_{2}$ levels were higher in group 2 than in group 1). Statistical analysis, however, could not demonstrate a significant difference due to this large variation among individual values.

\section{Conclusion}

Fetal cardiopulmonary bypass is feasible over short periods in sheep fetuses more than 0.85 gestational age with the surgical setting used in human neonatal cardiac surgery. Centrifugal and roller pumps are equally suitable. In younger fetuses, bleeding because of the fragility of atrial tissue remains a severe technical obstacle, and the centrifugal pump may cause atrial bleeding. However, the use of roller-head pump and the cannulation at atriocaval junction seem to provide better results.

Satisfactory pump flows were obtained using a centrifugalhead pump in the older fetuses. Nevertheless, total heart bypass was difficult to achieve in both old and young fetuses despite the use of adequate venous cannulae or different pump types. Further work is required to evaluate this finding because direct caval cannulation (which would provide total bypass) is not feasible in small fetuses because of the limited calibre of the vessel.

Younger fetuses showed a slow deterioration in gas status with partial recovery in most cases. The same deterioration was noted with umbilical flow, although to a lesser degree. Nevertheless, this study focused more on surgical feasibility than on the fetoplacental reaction to bypass. A specially designed flow probe would be necessary for the smaller fetuses.

Fetal body temperature was satisfactorily maintained during bypass, even in the absence of a heat-oxygenator exchanger. Amniotic fluid conservation was feasible. The anesthetic procedure was suitable and allowed for surgery without any overt signs of fetomaternal stress. Achievement of relative metabolic stability in terms of blood gas status, body temperature, and blood pressure warrants continuation of these experiments with the immediate aim of solving the problem of surgical bleeding in the young fetuses. Further work would be required to preserve fetoplacental function.

\section{Acknowledgments}

This work was supported by a grant from the Italian Ministry of Education and Research (MIUR) through the Fund CCCN2. The technical assistance of F. Bernini, L. Taddei, and D. Magnozzi from the Laboratory of Experimental Surgery (National Research Council and University of Pisa) is grateful acknowledged. We are also grateful to the Association 'Un cuore, Un Mondo' for their generous gift of a flowmeter. AC is a recipient of a graduate studentship of the Scuola Superiore S Anna.

\section{References}

1. Friedman AH, Kleinman CS, Copel JA: Diagnosis of cardiac defects: Where we've been, where we are and where we're going. Prenat Diagn 22: 280-284, 2002. 
2. Perolo A, Prandstraller D, Ghi T, et al: Diagnosis and management of fetal cardiac anomalies: 10 years of experience at a single institution. Ultrasound Obstet Gynecol 18: 615-618, 2001.

3. Allan LD, Apfel HD, Printz BF: Outcome after prenatal diagnosis of the hypoplastic left heart syndrome. Heart 79: 371-373, 1998.

4. Fraisse A, Nassi C, Sigaudy S, et al: Outcome of infants with hypoplastic left heart syndrome diagnosed in utero. Arch Mal Coeur Vaiss 95: 473-477, 2002.

5. Vandvik IH, Forde R: Ethical issues in parental decision-making: An interview study of mothers of children with hypoplastic left heart syndrome. Acta Paediatr 89: 1129-1133, 2000.

6. Kiserud T, Acharya G: The fetal circulation. Prenat Diagn 24: 1049-1059, 2004.

7. Tworetzky W, Marshall AC: Fetal interventions for cardiac defects. Pediatr Clin North Am 51: 1503-1513, 2004.

8. Kohl T, Sharland G, Allan LD: World experience of percutaneous ultrasound-guided balloon valvuloplasty in human fetuses with severe aortic valve obstruction. Am J Cardiol 85: 1230-1233, 2000.

9. Arzt W, Tulzer G, Aigner M, et al: Invasive intrauterine treatment of pulmonary atresia/intact ventricular septum with heart failure. Ultrasound Obstet Gynecol 21: 186-188, 2003.

10. Tulzer G, Arzt W, Franklin RC, et al: Fetal pulmonary valvuloplasty for critical pulmonary stenosis or atresia with intact septum. Lancet 360: 1567-1568, 2002.

11. Grigioni M, Carotti A, Daniele C, D'Avenio G, et al: Extracorporeal circulation in ewe's foetus: Towards a reliable foetal cardiac surgery protocol. A comparison of two cases. Int J Artif Organs 23: 189-198, 2000.

12. Reddy VM, Liddicoat JR, Klein JR, et al: Fetal cardiac bypass using an in-line axial flow pump to minimize extracorporeal surface and avoid priming volume. Ann Thorac Surg 62: 393-400, 1996.

13. Koong LJ, Garrett WN, Rattray PV: A description of the dynamics of fetal growth in sheep. J Anim Sci 41: 1065-1068, 1975.

14. Rudolph AM: Congenital Diseases of the Heart. Clinical Physiological Considerations. Armonk, NY, Futura Publishing, 2001. 